\title{
LETTERS
}

\section{Muddling Health Canada's powers and diluting accountability}

The opioid crisis is indeed serious, and there is an urgent need to propose and consider options for dealing with it. It is fair to suggest that Health Canada, as our regulator of drug safety, should play a major role in such considerations. ${ }^{1}$

The truth is, however, Health Canada has not served us well in the past in regard to drug safety. It does not help matters when the powers of our regulator to oversee drug safety are characterized incorrectly, as in this article. ${ }^{1}$ Although in 2014 Health Canada "spun" Vanessa's Law as giving it new powers to recall pharmaceuticals from the market, the truth is it always had the power to stop sales if there was even a whiff of a safety concern. ${ }^{2}$ The problem was that Health Canada rarely used this power.

There is a closely interconnected relationship between Health Canada and the pharmaceutical industry that calls for an indepth analysis of the culture within Health Canada and the political system within which it exists. To perpetuate the myth that Health Canada has had the power to take unsafe drugs off the market only since the introduction of Vanessa's Law in 2014 relieves the regulator of accountability for past regulatory failures and the unacceptable state of drug safety today.

\section{Pamela M. Forward RN MA}

Independent researcher and mediator, Mediation Place, Ottawa, Ont.

Cite as: CMAJ 2019 March 4;191:E266. doi: $10.1503 / \mathrm{cmaj} .71593$

\section{References}

1. Herder M, Juurlink D. High-strength opioid formulations: the case for a ministerial recall. CMAJ 2018;190:E1404-5.

2. Food and Drug Regulations (C.R.C., C. 870) [section C.01.013, C.08.006, C.08.003]. Available: https:// laws-lois.justice.gc.ca/eng/regulations/C.R.C.,_C._ 870/page-92.html (accessed 2018 Dec. 12).

Competing interests: None declared. 